\title{
Application of 3D Printing for Patient-Specific Silicone Stents: 1-Year Follow-Up on 2 Patients
}

\author{
Thomas R. Gildea ${ }^{a}$ Benjamin P. Young ${ }^{b}$ Michael S. Machuzak ${ }^{a}$ \\ ${ }^{a}$ Respiratory Institute, Cleveland Clinic, Cleveland, $\mathrm{OH}, \mathrm{USA} ;{ }^{\mathrm{b}}$ Department of Pulmonary, \\ Critical Care and Sleep Medicine, Case Western Reserve University, Cleveland, OH, USA
}

\section{Established Facts}

- Commercially available stents continue to be less than ideal in patients with complex benign airway disease. The impact of granulation, migration, infection, or biofilm limit the durable value of airway stents.

\section{Novel Insights}

- The use of patient-specific airway stents, combining the technology to make a 3-dimensional "prescription" and 3D printers, has been the topic of great enthusiasm for a subset of patients with very complex disease. This report is not only a report of a first-in-human experience but it also shows a durable effect over 1 year.

\section{Keywords}

Airway stent $\cdot$ Bronchoscopy $\cdot$ 3D printing

\begin{abstract}
Managing complex benign airway disease is a major challenge in interventional pulmonology. Airway stent placement can result in complications due to a variety of factors, including poor fit of the stent in the airway. We report the 1 -year outcome of 2 patients with airway disease caused by granulomatosis with polyangiitis (Wegener's) affecting the left main bronchus and secondary carina. These patients had
\end{abstract}

(C) 2018 S. Karger AG, Basel

\section{E-Mail karger@karger.com www.karger.com/res \\ KARGER}

not responded to systemic therapy or standard bronchoscopic techniques and had complications with commercially available airway stents. We describe a first-in-human, clinical experience to address these issues. Using computed tomography $(\mathrm{CT})$ imaging and 3D printing technology, we generated and implanted patient-specific silicone airway stents to address airway disease. 3D patient-specific stent prescription was created using a CT scan of the patient's chest and a

Aspects of the presented cases have been reported in meeting abstract form.

Thomas R. Gildea, MD, MS 
proprietary software package originally developed for orthopedic surgical planning. Silicone stents were manufactured and implanted. Observation for $>1$ year after implantation compared to the 6 months prior to patient-specific stent implant were compared for the number, clinically required stent changes, procedure time, and general clinical improvement per usual standard of care after airway stenting. Patients showed improved durability, a shorter procedure time, and improvement of patient-reported symptoms leading to a reduced need for stent changes and modifications. The use of 3D printing technology to make patient-specific silicone stents is feasible with early clinical proof of concept noting a durable improvement over 1 year of follow-up.

(c) 2018 S. Karger AG, Basel

\section{Introduction}

Complex nonmalignant airway disease is a major challenge. Airway stenting can restore luminal patency but is associated with complications due to challenges specific to airflow, cough, and secretion clearance. Limitations of available stent shapes, sizes, and materials have hindered improvements in airway stenting.

Silicone stents are the gold standard for inoperable, nonmalignant airway disease due to the ease of placement and removal with rigid bronchoscopy [1]. These have proven to be modifiable prior to implantation, which addresses some of the mechanical issues with "fit" [2]. However, challenges include infection, migration, mucus plugging, and granulation, necessitation of lifelong management, and recurrent stent replacement [3-6].

The last 3 decades have noted an evolution in stenting practices [7]. Self-expanding metallic stents have been used in successfully benign airway disease [8] but are associated with complications stemming from metal fatigue, granulation, and mucosal incorporation that can lead to inoperable conditions and resulted in an FDA "black box warning" in 2005 [8-10].

With the introduction of additive manufacturing in the medical field, patient-specific implants made from $3 \mathrm{D}$-printed molds are an innovative prospect for airway management [11-13]. The FDA has acknowledged the rise of $3 \mathrm{D}$ printing and recently released guidelines on the $3 \mathrm{D}$ printing of medical devices [14].

Here, we report the clinical application of a 3D-printing to make silicone $\mathrm{Y}$-stents to address the airway complexities of 2 patients. We describe the process of generating $3 \mathrm{D}$ prescription and using a $3 \mathrm{D}$ printer, a process for making patient-specific airway stents in patients failing

3D Printing and Patient-Specific Airway

Stents standard therapies. These stents were designed, manufactured, and implanted, with outcomes monitored for $>1$ year after implantation in this first-in-human project. Both patients retained their patient-specific stents for $>1$ year, with a reduced frequency of procedures and procedure time after implantation.

\section{Stent Manufacture}

The patients' chest computed tomography (CT) scan was imported into a proprietary software originally developed for orthopedic surgery (COS Inc., Cleveland, $\mathrm{OH}$, USA). A centerline for the airway was calculated, and the airway was digitally extracted and viewed as $3 \mathrm{D}$ rendering (Fig. 1). Based on this virtual model of each patient's anatomy, a "prescription" for the desired stent dimensions was defined that included the area, diameter, angulation, branching, length, and wall thickness (online supplementary Fig. 1; see www.karger.com/doi/10.1159/000489669 for all online suppl. material). The work of generating a $3 \mathrm{D}$ prescription is performed on a proprietary, web-based software program. Once the airway is segmented and displayed, the physician uses the software tools to place a series of spheres in the desired areas to adjust the contours and desired sizes to make a virtual representation based on clinical needs. External "studs," similar to those in existing silicone stents, were added to the stent design. Once the physician is satisfied with the design, it is approved for manufacture.

The mold for the prescribed stent was produced with $3 \mathrm{D}$ printing technology. Commercially available, medical grade silicone (Nusil Technology LLC, Carpinteria, CA, USA) was used to fill the mold. The stent was manufactured following controlled curing and washing processes, compliant with Code of Federal Regulations Title 21 of the FDA. The final stent was cleaned and finished for a smooth surface. The total manufacturing time required approximately 7 days in this project. The product is not commercially available in the United States. Prior to implantation, the stent was sterilized with standard steam sterilization on site. Lastly, the stent was repeatedly loaded and deployed via a standard stent deployment system (Lymol, Woburn, MA, USA) to assure durability.

\section{Procedure}

The existing airway stents were removed, and the airway was assessed under general anesthesia with rigid bronchoscopy and closed-circuit ventilation. The patient-specific implants were performed (Bryan-Lymol Stent Placement Kit) using standard instruments. Both patients were discharged the same day. 

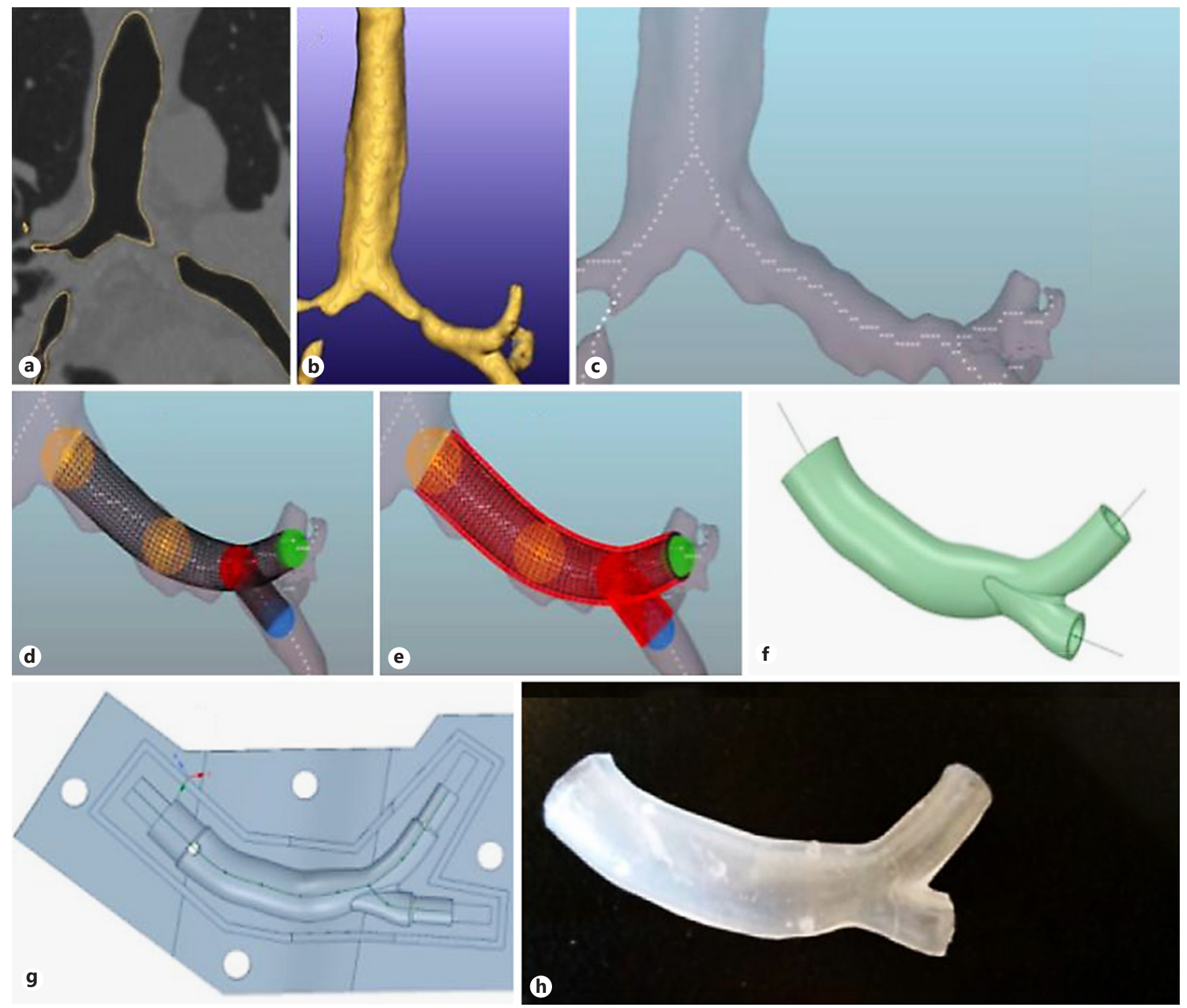

Fig. 1. Process of designing a patient-specific stent. a Coronal CT representation for segmentation. b $3 \mathrm{D}$ airway reconstruction, manually performed by the manufacturer. c Centerline plotting for stent alignment. $\mathbf{d}$ The spheres are placed and adjusted using the software tools to represent the diameter of the airway as any point desired by the physician. The red sphere is the join point that defines the volume of the carina of the Y-stent. e Proposed walls based on the defined stent wall thickness, and this can be adjusted.

f Final stent design prescription can be displayed with measurements and with the airway overlayed as desired in an interactive PDF file (Portable Document File). g Stent mold based on 3D design prescription. This is the file that is used for the $3 \mathrm{D}$ printed mold. The medical grade silicone is made from this mold since it is not possible to directly print medical grade silicone. The details of this process are propritary. $\mathbf{h}$ Final silicone Y-stent.

\section{Case Reports}

Case 1

A 56-year-old male with airway complications of granulomatosis with polyangiitis (Wegener's) had been managed in our practice since 2005. This patient was treated for debilitating respiratory obstructive symptoms and recurrent pneumonias for years utilizing

current bronchoscopic techniques, eventually requiring airway stenting for complex bilateral, multifocal airway disease. Previous procedures involved many variations of currently available stents with immediate benefit, yet he consistently suffered from stentrelated complications within only a few weeks or months of implantation. After years with various stents, granulation materialized at the ends of the stents where imprecise fit occurs and was 

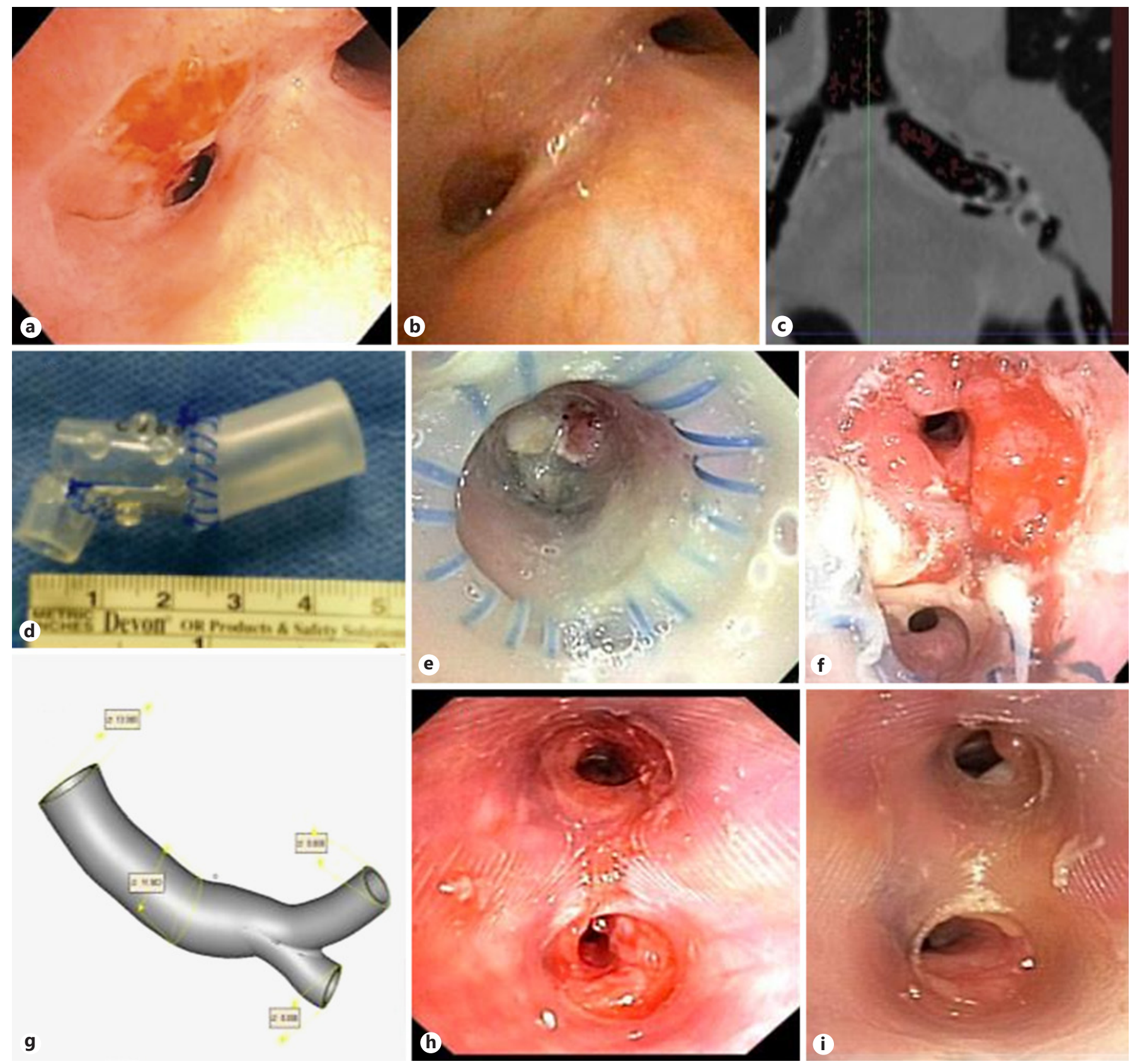

Fig. 2. Radiographic and airway images of Patient 1. a Main carina with strictures of the bilateral main stent bronchi prior to any of the interventions 4 years prior to customized stent implantation. b Left secondary carina showing complex airway strictures despite balloon dilations, medical management. Note the scar and splayed carina and severe narrowing of the left upper and lower divisions. c Coronal CT radiograph showing the multiple levels of stenosis at the orifice of the left main bronchus and the secondary carina despite hand-sewn customized stenting for the left lower lobe. d A manually-sewn customized stent for complex stenosis and curvature of the left main bronchus and secondary carina. e Left main

bronchus with mid-portion anastomosis. $\mathbf{f}$ Bronchoscopic image of the left main bronchus to the left secondary carina with a "customized" silicone stent with extension sewn to the stent in the lower division. Note the severe granulation around the upper division where another stent had been but migrated out. g 3D prescription of the left main and secondary carina Y-stent. $\mathbf{h}$ Patient-specific $\mathrm{Y}$-stent in the left main bronchus and secondary carina on the day of patient-specific stent implantation. i Patient-specific stent 13 months after implantation. Note 1 tiny nodule of granulation in the upper division and reduction of the mucosal inflammation in the lower division. 

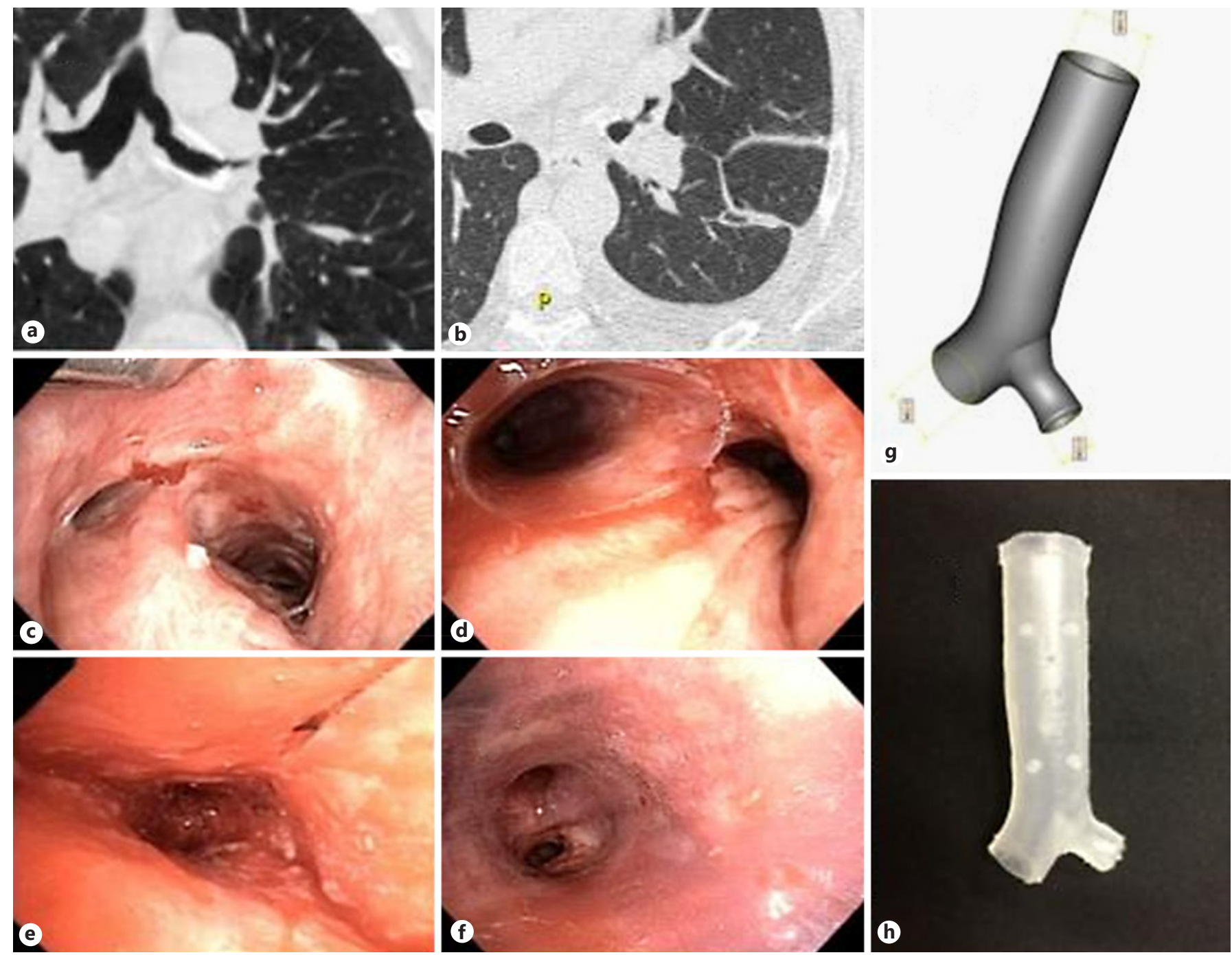

Fig. 3. Radiographic and airway images of Patient 2. a Coronal CT chest with the original metallic stent incorporated into the left main bronchus airway wall with in-stent stenosis. b Axial chest CT with stenosis in the left upper division. c Main carina with recurrent stenosis after original stent extraction. $\mathbf{d}$ Patient-specific stent in the left main bronchus. e Distal left main bronchus and secondary carina. $\mathbf{f}$ Distal left main bronchus with a patient-specific Ystent. g 3D prescription stent. h Patient-specific Y-stent for the left main bronchus. associated with clinical infection, as the patient became colonized with Pseudomonas aeruginosa. A stent with a "cinch" was designed and implanted in the bronchus intermedius, achieving durable results after much experimentation. The left main bronchus was characterized by a combination of strictures and malacia. After multiple failed treatment attempts using standardized stents, customized modifications to the silicone stent showed promise, but still lacked durability and ideal fit. Ultimately, a stent was crafted for the left bronchus by sewing together several silicone stents over various sizes and angles to achieve a left main bronchus carina stent, hybridized with a fully covered left upper division metal stent. Several attempts of being stent free were also not tolerated.

In the 6 months prior to implanting the patient-specific stent, the patient underwent some variation of therapeutic bronchosco- py every 39 days on average, with procedure times averaging 59 min each, resulting in an average stent life of 52 days.

Working with the Cleveland Clinic IRB and FDA, a compassionate use exemption was submitted that described the clinical need and manufacturing (Fig. 2) for the investigational device based on expanded access FDA process [15]. Clearance to use this device in this patient was received in January 2016.

Forty-eight hours after implantation, the patient had increased secretions and obstructive symptoms. He returned for bronchoscopy and stent assessment. The stent was coated with copious thin secretions, residual polypoid granulation from the prior procedure was noted occluding the distal ends of the stent that was easily removed. The patient's symptoms immediately improved, and he was discharged the same day. Bronchial washing cultures from the 
initial implant procedure grew Pseudomonas; oral and inhaled antibiotics were initiated, and the infectious symptoms improved.

At the 4-month follow-up, a CT scan of the chest showed evidence of regression of the airway inflammation around the stent in the left main bronchus. With the left main bronchus significantly improved, medical treatment shifted to the right side of the airway, where stenosis and granulation were observed around the modified standard silicone stent that was placed in a prior procedure. This was managed with balloon bronchoplasty, cryotherapy, and steroid injection, with symptomatic improvement observed immediately upon awakening from anesthesia.

After implantation, the patient had procedures every 135 days (vs. 39 days before implantation) on average, and the stent remained implanted for 405 days before being replaced per the protocol submitted to the FDA.

\section{Case 2}

A 45-year-old female with airway complications of granulomatosis with polyangiitis (Wegener's) had been referred for management of complications associated with an existing embedded metallic stent in her left main bronchus in 2013. At the time of initial presentation, she was having bronchoscopy procedure every 2 weeks to clean out her airway. She had been treated with methotrexate and rituximab. Three successive therapeutic procedures were required to remove the existing metal stent and for the transition to a commercially available silicone stent. Over the next 18 months, she underwent multiple procedures to manage the complications of poor fit associated with difficult angulation and differing diameter of the proximal and distal left main bronchus. A custom stent was ordered from Novatech SA (Voie Antiope, France) but was unsuccessful because the stent was too thick/bulky at the Y-configuration connection for successful implantation.

Within the 6 months prior to the placement of the patientspecific stent, the patient underwent bronchoscopy every 23 days on average, with an average procedure time of $45 \mathrm{~min}$ and an average stent life of 51 days. Clearance to use this device was received in April 2016.

The patient has had an uneventful course, with no unplanned procedures since implantation of the patient-specific stent. She has been able to increase the time between her procedures (every 42 days on average) and slightly decrease the procedure time (42 min on average) owing to the presence of less inflammation and scar and granulation tissue. Within the 18 months preceding the patient-specific implant, the patient had 7 stents placed and removed (related to stent complications) and, since the placement of the patient-specific stent, she has had the same stent in place for over 12 months (Fig. 3). The original patient-specific stent was replaced with a new stent prescription to address stenosis and granulation in an adjacent orifice of the left lower lobe under another expanded access request.

\section{Discussion}

The use of airway stenting in complex benign airway disease is fraught with complications, with no viable tracheal or bronchial replacement currently available despite early reports [16]. Airway devices should be considered only when all other options have been exhausted. In most cases, surgical correction is favored, yet given the systemic and bilateral nature of the disease, history of chronic infections, and the well-functioning lung parenchymal of the 2 patients described in this report, transplant and other surgical interventions were not viable options. The cases described herein highlight the broad range of clinical challenges with stenting. Systemic anti-inflammatory treatments and standard endobronchial therapy did not resolve the stricture or reduce inflammation to prevent severe obstructive symptoms of recurrent infections. Stenting, while immediately helpful, eventually results in problems associated with foreign body reaction to mechanical stress, as well as the risk of recurrent infection, all of which shorten the life of the stent. The ability to customize or order custom stents has been reported. However, it is still challenging to fashion devices that work well in all patients, as exemplified by the lack of sustained benefits in both patients described in this report.

While 3D printing has been used to create airway splints and T-tubes [17-19], this is the first case of an implantable bronchial stent with FDA clearance for compassionate use in benign airway disease using an approved, but modified, proprietary software package and commercially available materials.

In these cases, we removed the stent after 1 year based on the recommendations for standard silicone stents and in accordance with our FDA request. Both patients may have been able to maintain the stents longer; however, assessment after 1 year served as an opportunity to evaluate whether changing the stent prescription could improve upon the initial success. Based on the results in these 2 patients, patient-specific airway stenting appears to be a viable long-term option in individuals with complex benign airway disease.

There remain myriad challenges in the clinical practice of patient-specific implants. It is not clear in whom these stents will be most useful. We suspect that it may be as many as $30 \%$ of our practice of patients with benign airway disease and most likely all patients with Y-stents. The technology for imaging airway disease is imperfect, given the dynamic changes in the airway related to respiration. The ability to generate a $3 \mathrm{D}$ prescription requires intimate knowledge of the disease and predict the ability to work with software to translate clinical judgment to a device. Furthermore, as infection and mucus adherence is still not fully addressed by shape alone. This first-in-human, proof-of-concept report supports that patient-specific stents are effective in patients with benign airway disease paves the way for further definitive investigation. 


\section{Acknowledgements}

Keith Grafmeyer and Jacob Eva from COS supported the communication with FDA and the design manufacturing of the stent. Drs. Francisco Almeida and Sonali Sethi have participated in the care of the patients. NIH-NCAI grant support (Grant 1U54HL119810-03) was given.

\section{Statement of Ethics}

The authors affirm that the work was performed with oversight of the Cleveland Clinic IRB and the United States Food and Drug Administration Clearance with compassionate use as described with patients' signed informed consent.

\section{Disclosure Statement}

The authors have no conflicts. Cleveland Clinic and Cleveland Clinic Institutional Officials/Leaders have an equity interest in Custom Orthopaedic Solutions and are entitled to royalty payments from the company for technology developed at Cleveland Clinic. Custom Orthopaedic Solutions is the manufacturer of the stents.

\section{References}

1 Dumon JF: A dedicated tracheobronchial stent. Chest 1990;97:328-332.

2 Breen DP, Dutau H: On-site customization of silicone stents: towards optimal palliation of complex airway conditions. Respiration 2009; 77:447-453.

3 Dutau H, Cavailles A, Sakr L, et al: A retrospective study of silicone stent placement for management of anastomotic airway complications in lung transplant recipients: shortand long-term outcomes. J Heart Lung Transplant 2010;29:658-664.

4 Wood DE, Liu YH, Vallieres E, Karmy-Jones R, Mulligan MS: Airway stenting for malignant and benign tracheobronchial stenosis. Ann Thorac Surg 2003;76:167-172; discussion 173-174.

5 Casal RF: Update in airway stents. Curr Opin Pulm Med 2010;16:321-328.

6 U.S. Food and Drug Administration, Center for Devices and Radiological Health: FDA public health notification: complications from metallic tracheal stents in patients with benign airway disorders. August 2, 2005. www.jsre.org/info/0801_fda.pdf.
7 Dutau H, Breen D, Bugalho A, et al: Current practice of airway stenting in the adult population in Europe: a survey of the European Association of Bronchology and Interventional Pulmonology (EABIP). Respiration 2018;95: 44-54.

8 Saad CP, Murthy S, Krizmanich G, Mehta AC: Self-expandable metallic airway stents and flexible bronchoscopy: long-term outcomes analysis. Chest 2003;124:1993-1999.

9 Fortin M, MacEachern P, Hergott CA, Chee A, Dumoulin E, Tremblay A: Self-expandable metallic stents in nonmalignant large airway disease. Can Respir J 2015;22:235-236.

10 Chung FT, Lin SM, Chen HC, et al: Factors leading to tracheobronchial self-expandable metallic stent fracture. J Thorac Cardiovasc Surg 2008;136:1328-1335.

11 Trevisan F, Calignano F, Aversa A, et al: Additive manufacturing of titanium alloys in the biomedical field: processes, properties and applications. J Appl Biomater Funct Mater 2018;16:57-67.

12 Morrison RJ, Hollister SJ, Niedner MF, et al: Mitigation of tracheobronchomalacia with 3D-printed personalized medical devices in pediatric patients. Sci Transl Med 2015;7: 285 ra64.
13 van Eijnatten M, van Dijk R, Dobbe J, Streekstra G, Koivisto J, Wolff J: CT image segmentation methods for bone used in medical additive manufacturing. Med Eng Phys 2018;51: 6-16.

14 FDA: 3D printing of medical devices. 2018

15 FDA: Expanded access (sometimes called "compassionate use"). 2018.

16 Delaere P, Vranckx J, Verleden G, De Leyn P, Van Raemdonck D, Leuven Tracheal Transplant Group: Tracheal allotransplantation after withdrawal of immunosuppressive therapy. N Engl J Med 2010;362:138-145.

17 Cheng GZ, Folch E, Brik R, et al: Three-dimensional modeled $\mathrm{T}$-tube design and insertion in a patient with tracheal dehiscence. Chest 2015;148:e106-e108.

18 Zopf DA, Hollister SJ, Nelson ME, Ohye RG, Green GE: Bioresorbable airway splint created with a three-dimensional printer. N Engl J Med 2013;368:2043-2045.

19 Guibert N, Didier A, Moreno B, et al: Treatment of post-transplant complex airway Stenosis with a three-dimensional, computer-assisted customized airway stent. Am J Respir Crit Care Med 2017;195:e31-e33. 